\title{
Tevatron Highlights
}

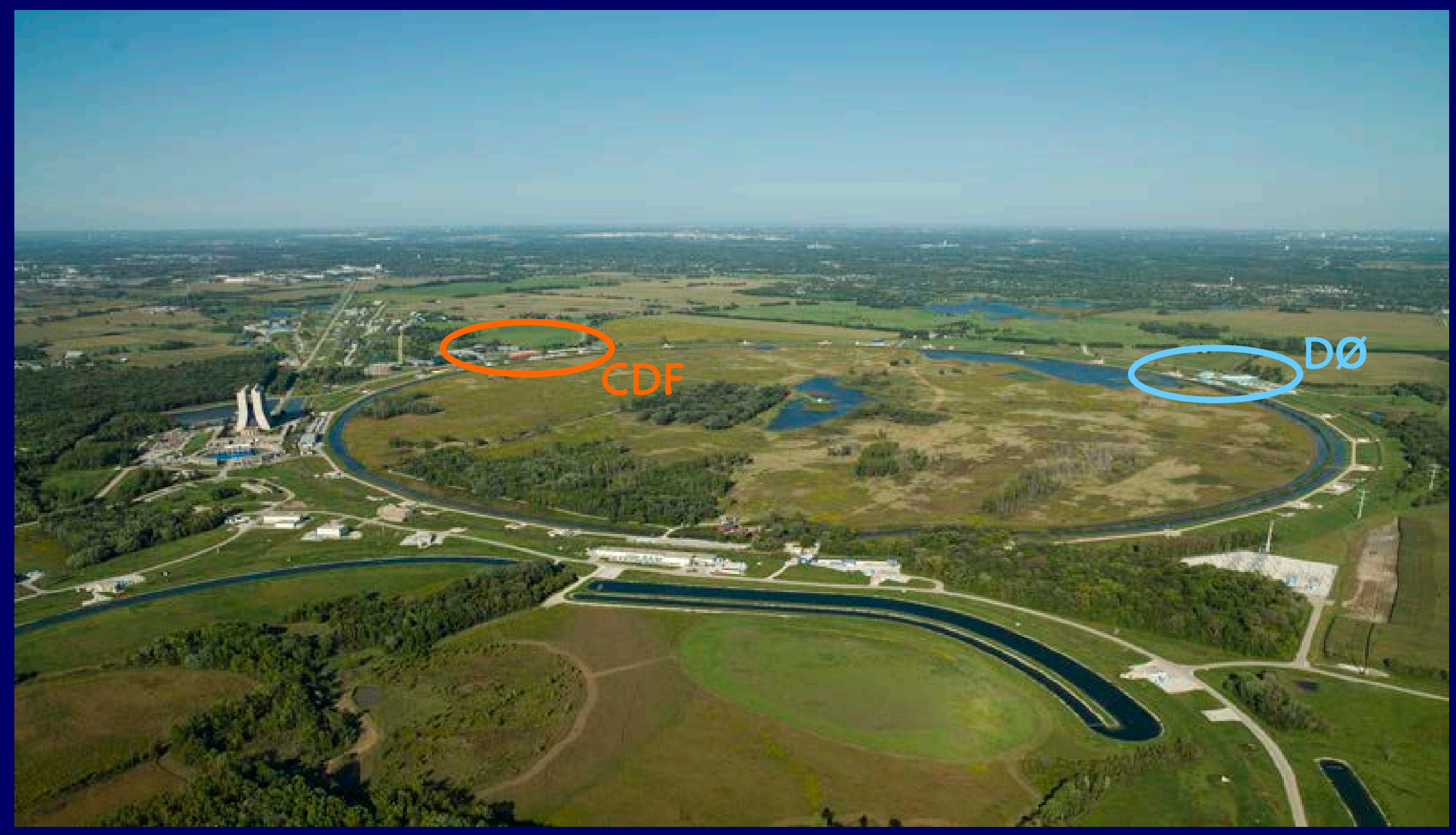

Fermilab Users Meeting P. Grannis, June 21, 2018 for the CDF and DO Collaborations
This document was prepared by [CDF and DO Collaborations] using the resources of the Fermi National Accelerator Laboratory (Fermilab), a U.S. Department of Energy, Office of Science, HEP User Facility. Fermilab is managed by Fermi Research Alliance, LLC (FRA), acting under Contract No. DE-AC02-07CH11359. 


\section{The Tevatron Collider}

Run 01987 - 1988: 1.8 TeV, CDF only, $4 \mathrm{pb}^{-1}$

Run I 1992 - 1996: 1.8 TeV, CDF+D0: $120 \mathrm{pb}^{-1}$

Run II 2001 - 2011: 1.96 TeV, $12 \mathrm{fb}^{-1}$. Added Main Injector, Recycler, helical orbits, magnet alignment ...

Max. Instantaneous $L \approx 4.3 \times 10^{32}$ $\mathrm{cm}^{-2} \mathrm{~s}^{-1}$ (30M collisions/s)

The superb performance of the Tevatron complex was the foundation for the physics accomplishments of CDF and DO. We are indebted to the scientists and engineers of the Accelerator Division.
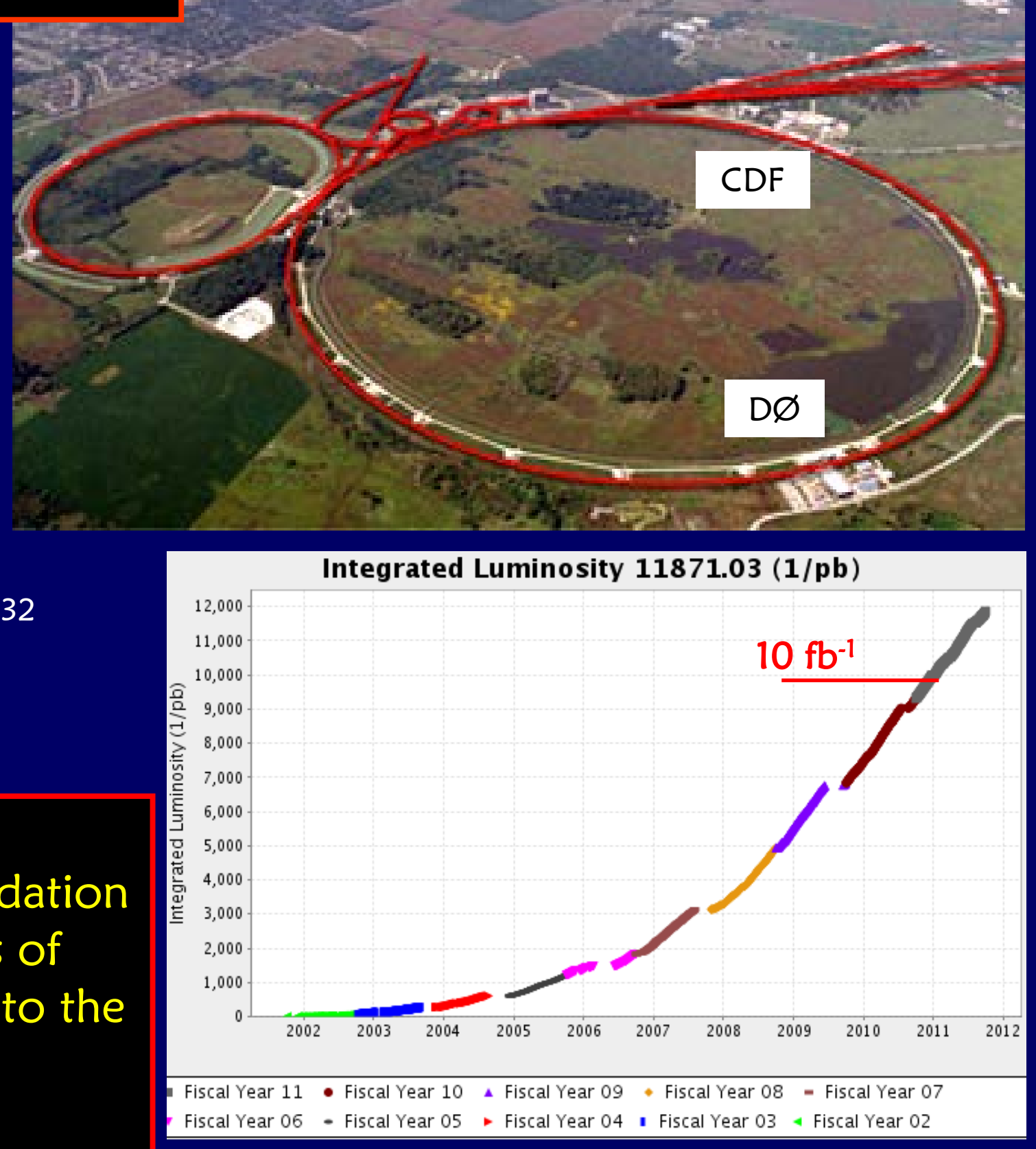


\section{The experiments}
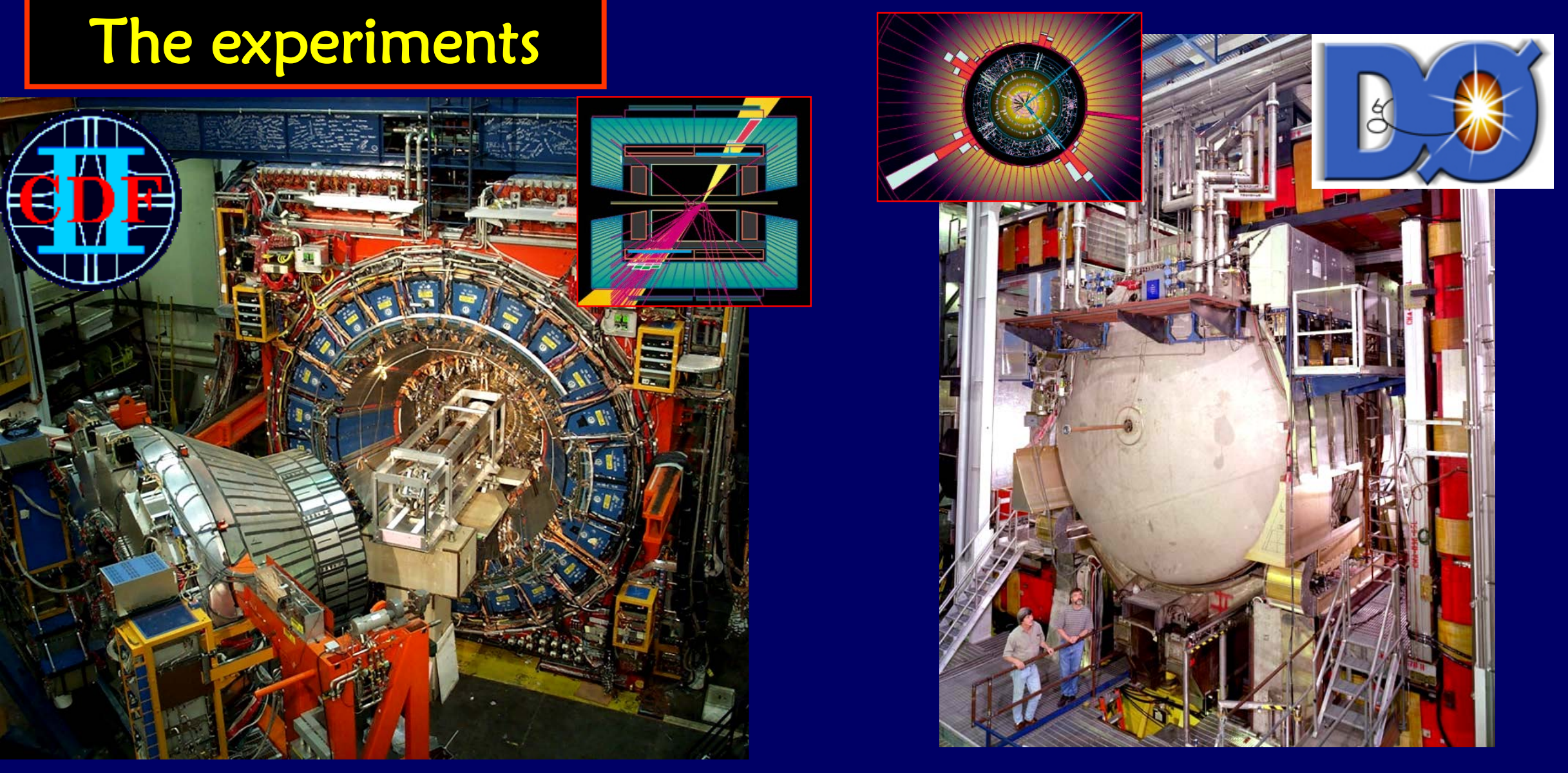

CDF and D $\varnothing$ were quite different in Run I. Major detector upgrades for Run II: CDF: new tracker, new Si vertex det, upgraded forward calorimeter and muons DØ: add solenoid, fiber tracker, Si vertex and preshower detectors, new forward muon detectors.

The upgraded experiments looked more like each other, but still with complementary strengths. Cross checks with $>1$ experiment were crucial! 


\section{Publications since Users Meeting 2016}

(No report in 2017 due to Fermilab's 50th $)$

1. Search for fermiophobic Higgs: Phys. Rev. D 93, 112010 (2016)

2. WW and WZ XSs with $\ell^{ \pm}$and heavy flavor jets: Phys. Rev. D 94, 032008 (2016).

3. Spin correlation between top and antitop: Phys. Lett. B 757, 199 (2016).

4. $B_{s}{ }^{0}$ lifetime in the $C P$-odd $B_{s} \rightarrow J / \psi f_{0}(980)$ : Phys. Rev. D 94, 012001 (2016).

5. Evidence for a $B_{s} \pi$ state: Phys. Rev. Lett. 117, 022003 (2016),

6. Top mass using matrix element method in dileptons: Phys. Rev. D 94 , 032004 (2016).

7. Inclusive ttbar XS and top quark pole mass: Phys. Rev. D 94, 092004 (2016).

8. Top quark polarization in ttbar lepton + jets: Phys. Rev. D 95, 011101(R) (2017).

9. Direct CPV charge asymmetry in $B^{ \pm} \rightarrow \mu^{ \pm} v_{\mu} D^{0}$ : Phys. Rev. D 95, 031101(R) (2017).

CDF

10. $\mathrm{D}^{+}$meson cross section at low $\mathrm{p}_{\mathrm{T}}$ : Phys. Rev. D 95, 092006 (2017).

$*$ CDF pub.\# 700

11. Combination of D0 measurements of top mass: Phys. Rev. D 95, 112004 (2017).

DO

12. Observation of $\mathrm{Y}(4140)$ in $\mathrm{B}^{ \pm} \rightarrow \mathrm{J} / \psi \phi \pi \mathrm{K}$ decays, Mod. Phys. Lett. A32, 1750139 (2017)

13. Inclusive Isolated prompt photon cross section: Phys . Rev. D 96, 092003 (2017).

CDF

14. Combined F-B asymmetry in ttbar production: Phys. Rev. Lett. 120, 042001 (2018).

15. Search for exotic meson X(5568): Phys. Rev. Lett. 120, 202006 (2018).

$\mathrm{CDF}+\mathrm{DO}$

16. Study of $X(5568) \rightarrow B s \pi$ in semileptonic Bs decays: Phys. Rev. D 97, 092004 (2018).

17. Effective weak mixing angle in $Z \rightarrow \ell^{+} \ell^{-}$: Phys. Rev. Lett. 120, 241802

18. Tevatron combination of $\sin ^{2} \theta_{\text {eff }}$ lept: Phys. Rev. D, in press (2018). ).

CDF

DO

DO

$C D F+D O$ 


\section{Publications since Users Meeting 2016}

The Tevatron results over the past two years still represent $40 \%$ of the physics papers based on Fermilab accelerator operations. (The rest are almost all neutrino cross sections and oscillation measurements.)

Even during the LHC era, the Tevatron papers have added significantly to our understanding of:

$>$ QCD

$>$ Heavy flavor physics

$>$ Electroweak interactions

$>$ Top quark

$>$ Higgs boson

$>\quad$ (but very little to searches for new phenomena! )

The highlights to follow cover some of the Tevatron legacy results, as well as some since the last Users' meeting report (marked

We have benefitted greatly from the Computing Division's support of the CDF and DO hardware platforms and software systems, particularly in keeping our aging systems going. 


\section{Highlights - Heavy Flavor}

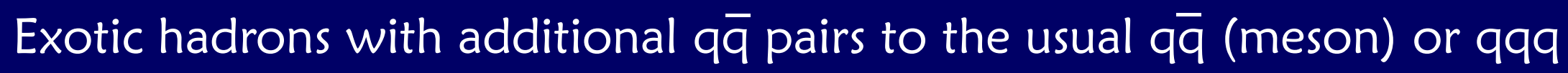
(baryon) have long been predicted but only recently seen, both in $\mathrm{e}^{+} e^{-}$and hadron collisions. Those with heavy flavor are easier to identify than purely light quark exotics due to distinctive decays and lower backgrounds. CDF and DO have added important new information on exotics' production mechanisms (e.g. * prompt vs. in decay products).

** In 2016 D0 published strong evidence for a new state $\mathrm{X}(5568)^{+} \rightarrow \mathrm{B}_{s}^{0} \pi^{+}$with $\mathrm{B}_{s}{ }^{0} \rightarrow \mathrm{J} / \psi \phi$. The minimal quark content is bsu $\bar{d}$ - the first exotic state with 4 quark flavors. LHC experiments did not see it in pp collisions.

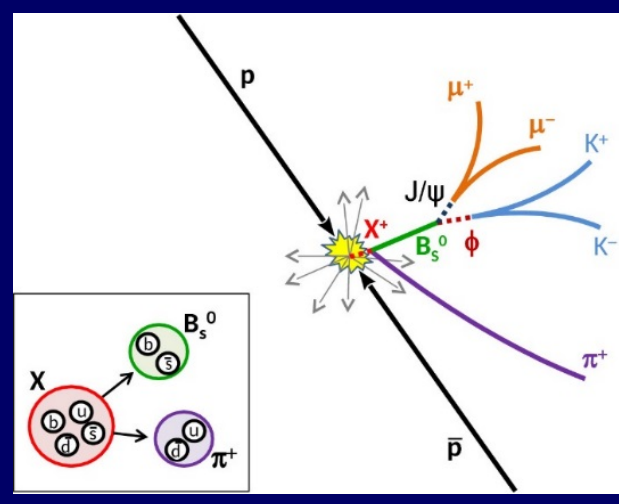

$* *$ In 2018, CDF did not observe X(5568) in $\mathrm{B}_{s}{ }^{0} \rightarrow \mathrm{J} / \psi \phi$

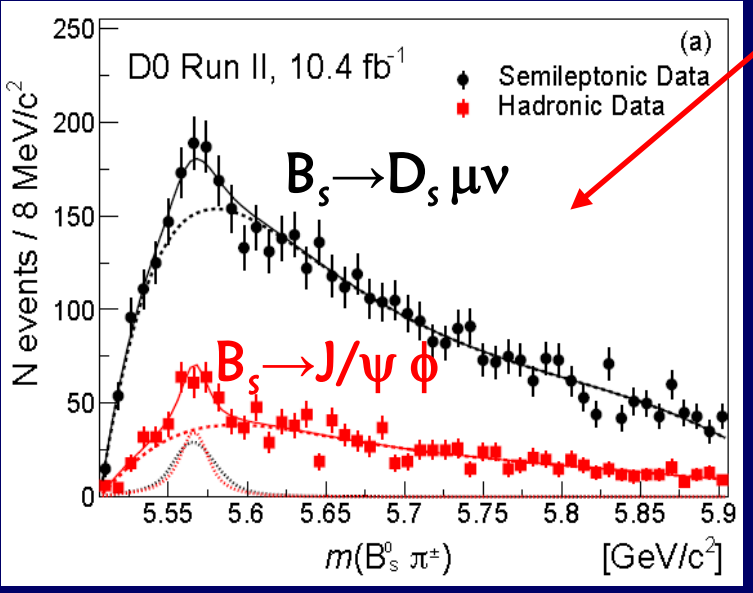
but ** DO did see it in $\mathrm{B}_{s}^{0} \rightarrow \mathrm{D}_{s}^{+} \mu^{-} v$. A combined fit of the two channels gives significance $=6.7 \sigma$.

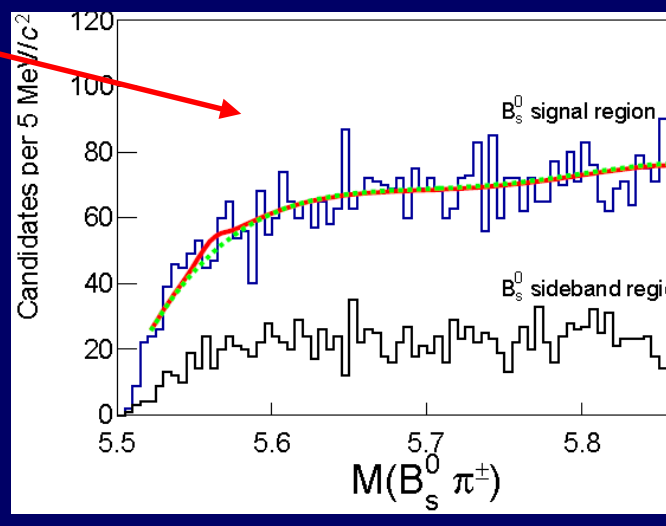

A puzzling situation: D0 signal comes primarily when at least one $\mu$ from $\mathrm{J} / \psi$ is outside CDF coverage. 


\section{Highlights - Electroweak}

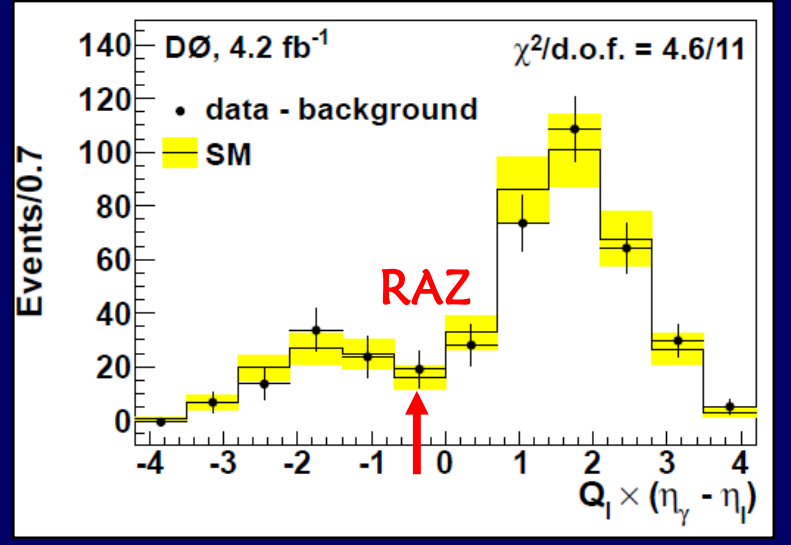

W boson mass (CDF+D0) measured $16 \mathrm{MeV}(0.02 \%)$ uncertainty - one of the most powerful tests of the EW sector of the Standard Model.

Many measurements of vector boson trilinear couplings. Here, the first observation of Radiation Amplitude

Zero in WWV coupling due to interference of $\mathrm{s}$ - and t-channel processes.

** (2018, PRD in press): Measure the weak mixing angle that governs EWSB using the $Z \rightarrow \ell^{+} \ell^{-}$F-B asymmetry. Combined Tevatron result ( $\delta \sin ^{2} \theta_{\text {eff }}=0.00033$ ) rivals the precision of 20-year old LEP-1 and SLD measurements $(\delta=0.00029$ and $\delta=0.00026)$ and is midway between them, and also in excellent agreement with world average.

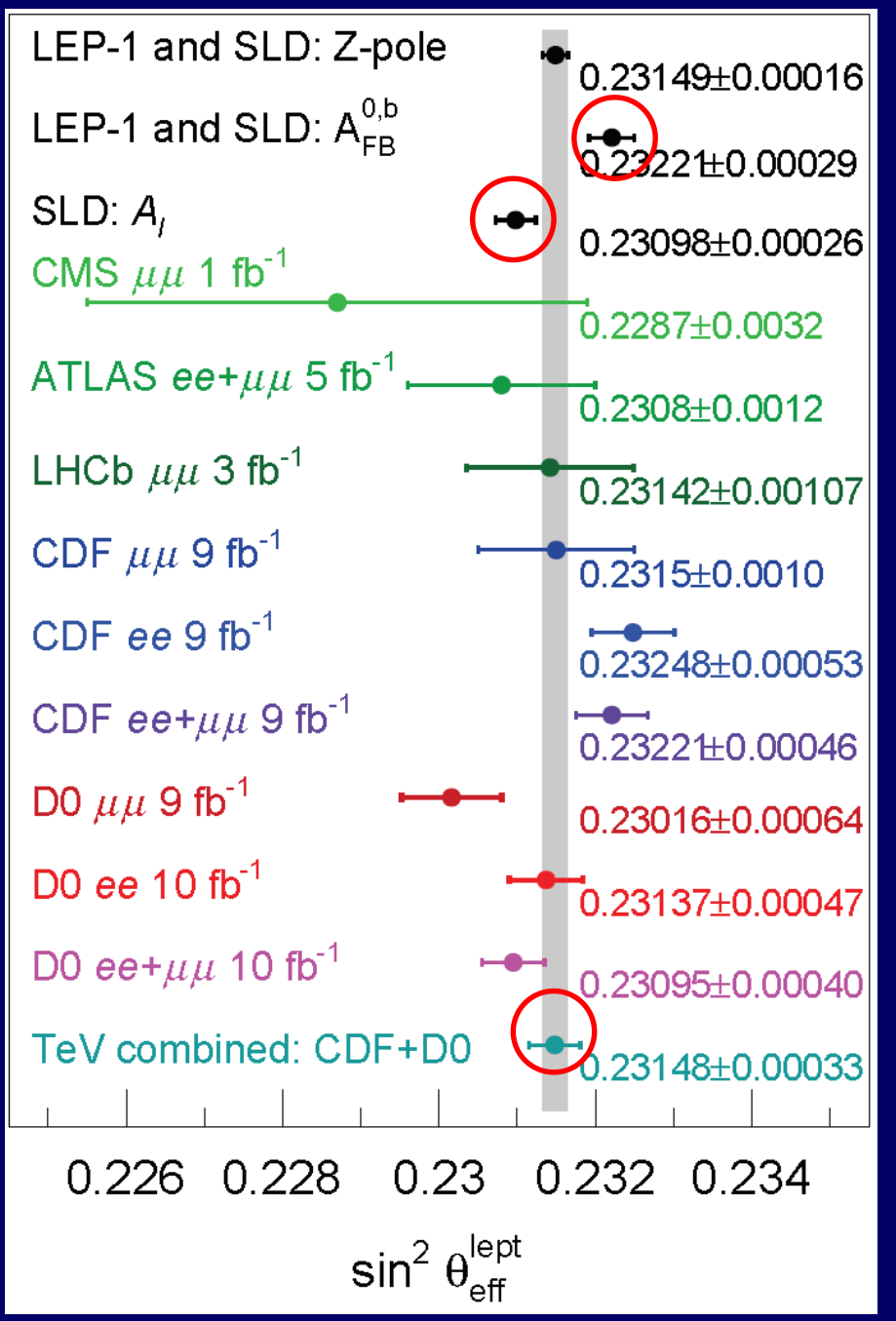




\section{Highlights - Top Quark}

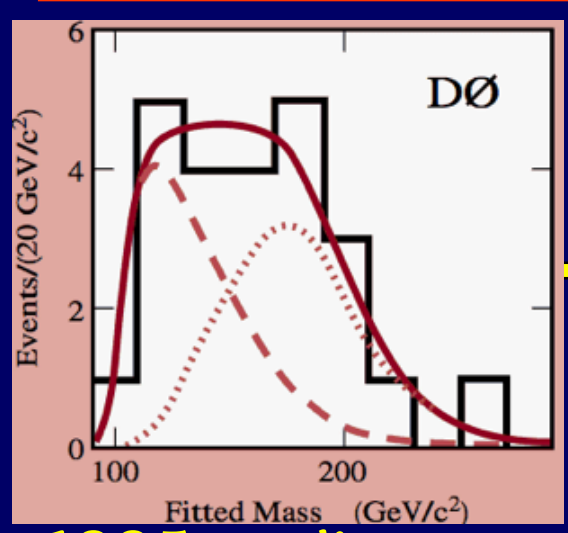

1995 top quark discovery by CDF and DO was the most notable Tevatron result

1995 at discovery (13 D0 tt events)

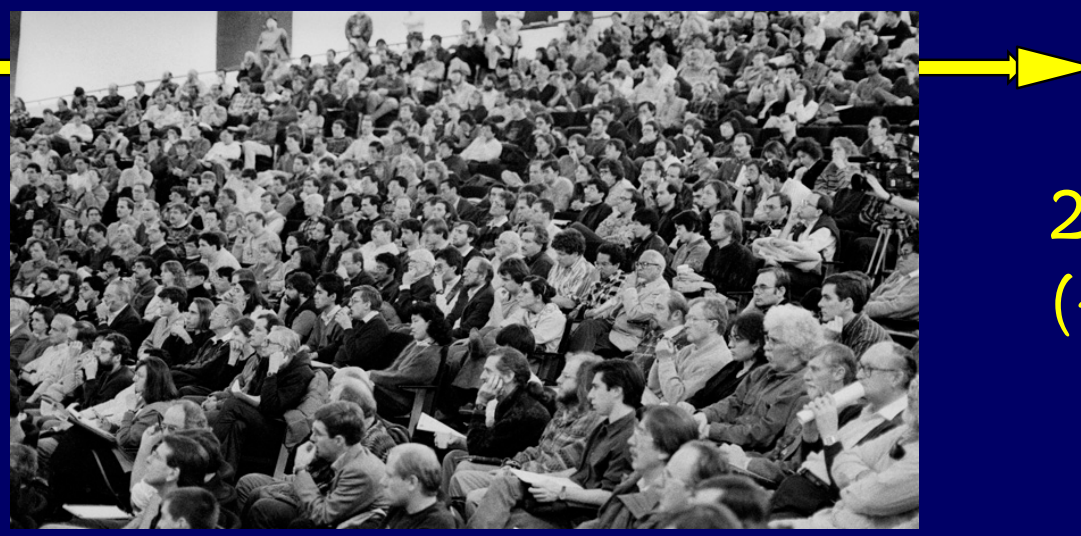

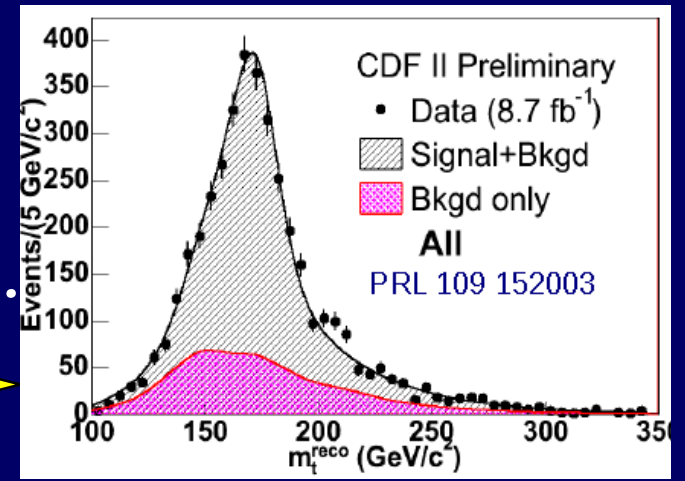

2013 near final $\int \mathcal{L} d t$ ( $\sim 3000$ CDF tt events)

Early measurements of forward-backward tt asymmetries showed excess over SM prediction both vs. $m_{t t}$ and $y_{t t}$, suggesting possible non-SM physics.

** Recent combination of final CDF and DO measurements agree with new Standard Model NNLO(QCD) $+N L O$ (EW) theory.
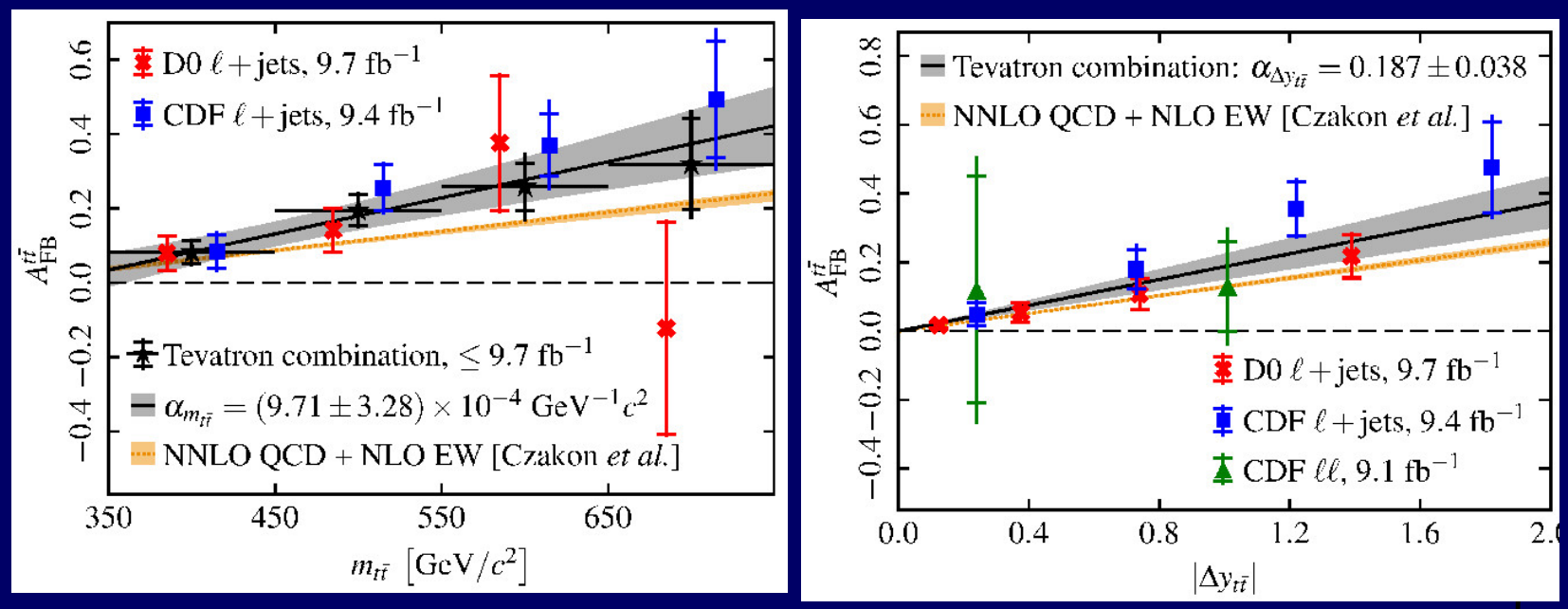


\section{Highlights - Top Quark}

The fact that top decays before hadronizing allows measurements of top charge, polarization, spin correlations, lifetime, CPT violation, decay W helicity, and searches for FCNC, resonances, anomalous couplings etc.

* DO combination of top mass using comparison to MC templates (based on the matrix element method) for all channels: $m_{t}=174.95 \pm 0.75 \mathrm{GeV}(0.4 \%)$ (CDF analysis is in progress).

Also measure theoretically well-defined top pole mass by comparing measured $\sigma_{\text {tot }}$ with $\mathrm{m}_{\mathrm{t}}$-dependent QCD NNLO/NLL calculations.

Single top quark production via EW reactions was first discovered in 2009. Both s- and t-channel W exchange processes were observed.
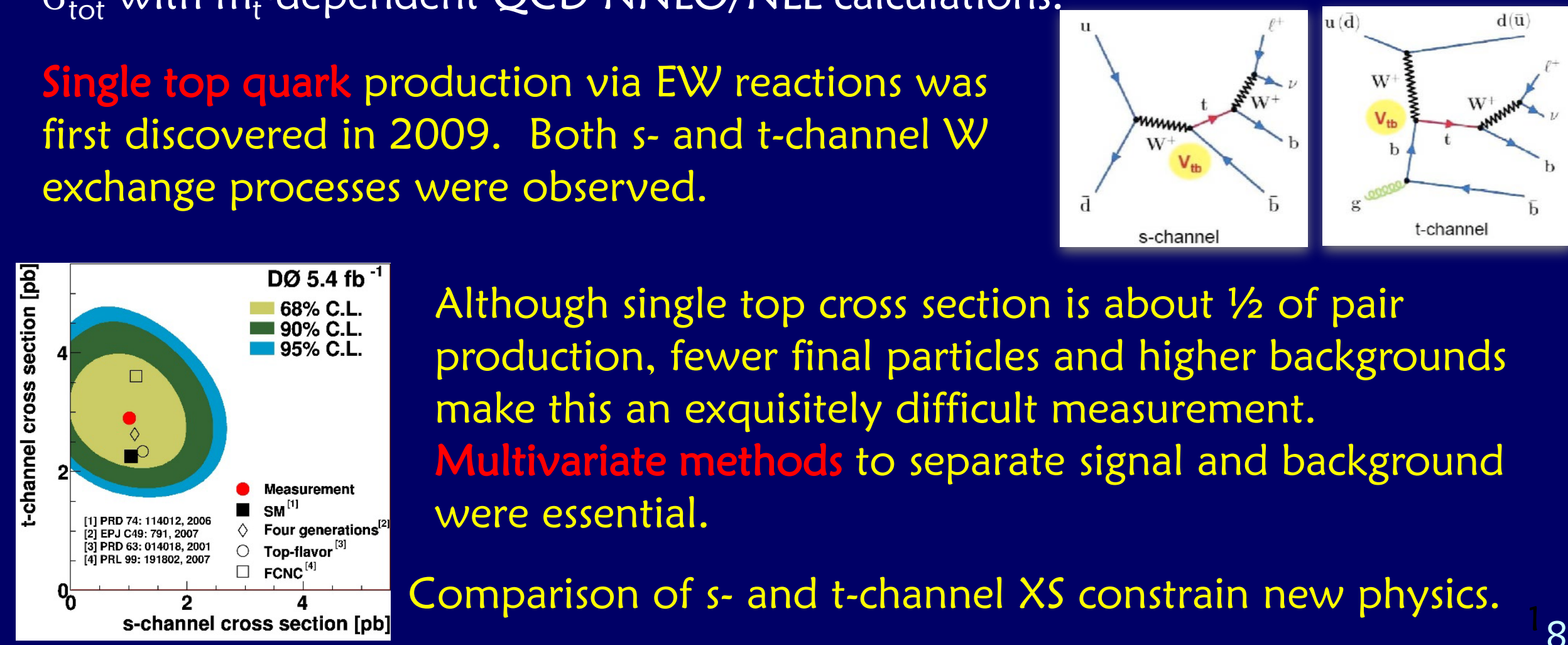

Although single top cross section is about $1 / 2$ of pair production, fewer final particles and higher backgrounds make this an exquisitely difficult measurement. Multivariate methods to separate signal and background were essential.

Comparison of s-and t-channel XS constrain new physics. 


\section{Highlights - New phenomena}

LHC has overtaken Tevatron in almost all aspects of searches

LHC squark gluino limits (already in 2011)

Tevatron squark gluino limits

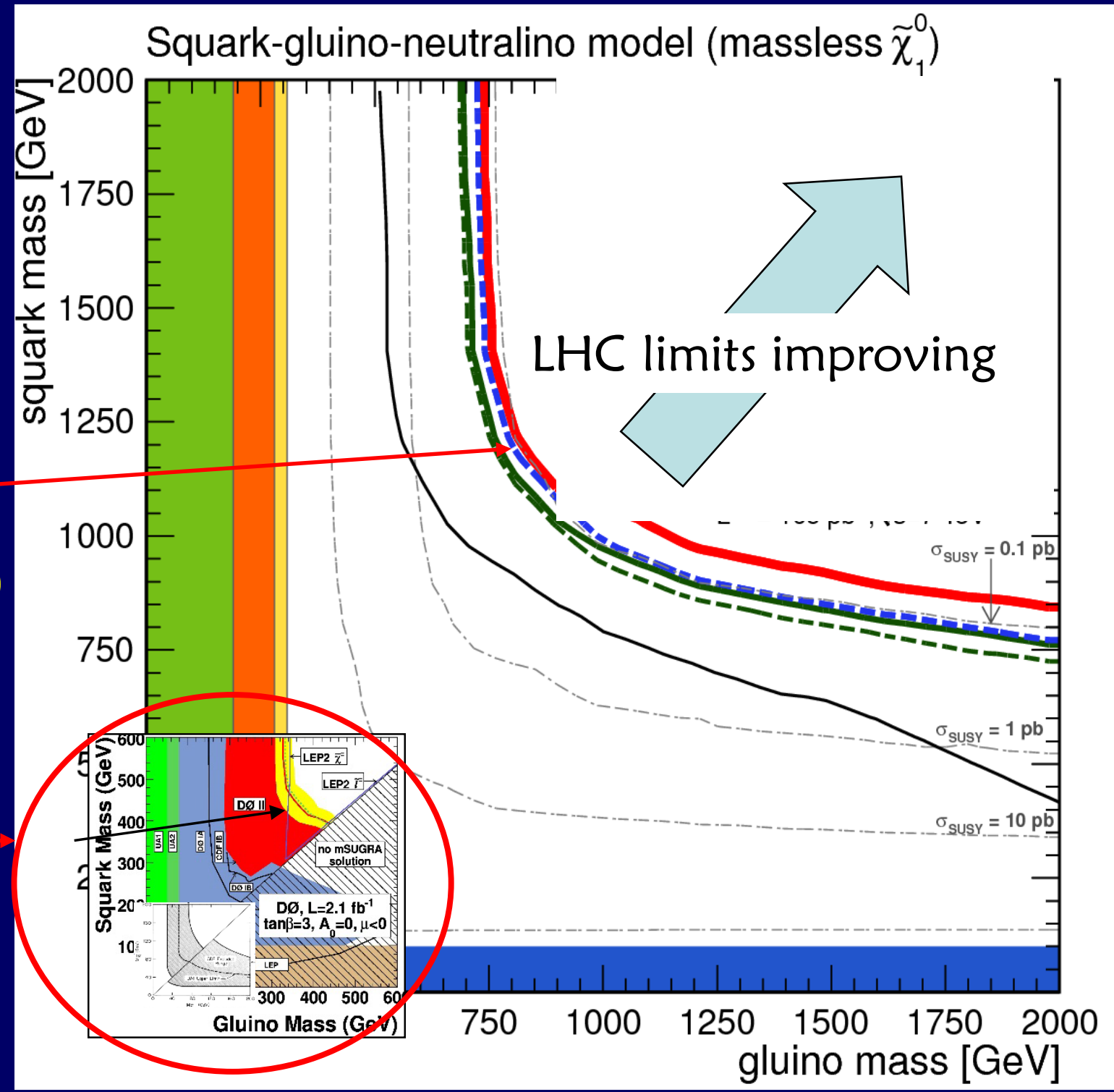




\section{Highlights - New phenomena}

LHC has overtaken Tevatron in more ways than one

\section{"400 Physicists} Fail to Find Supersymmetry" (NYTimes, ca 1992)

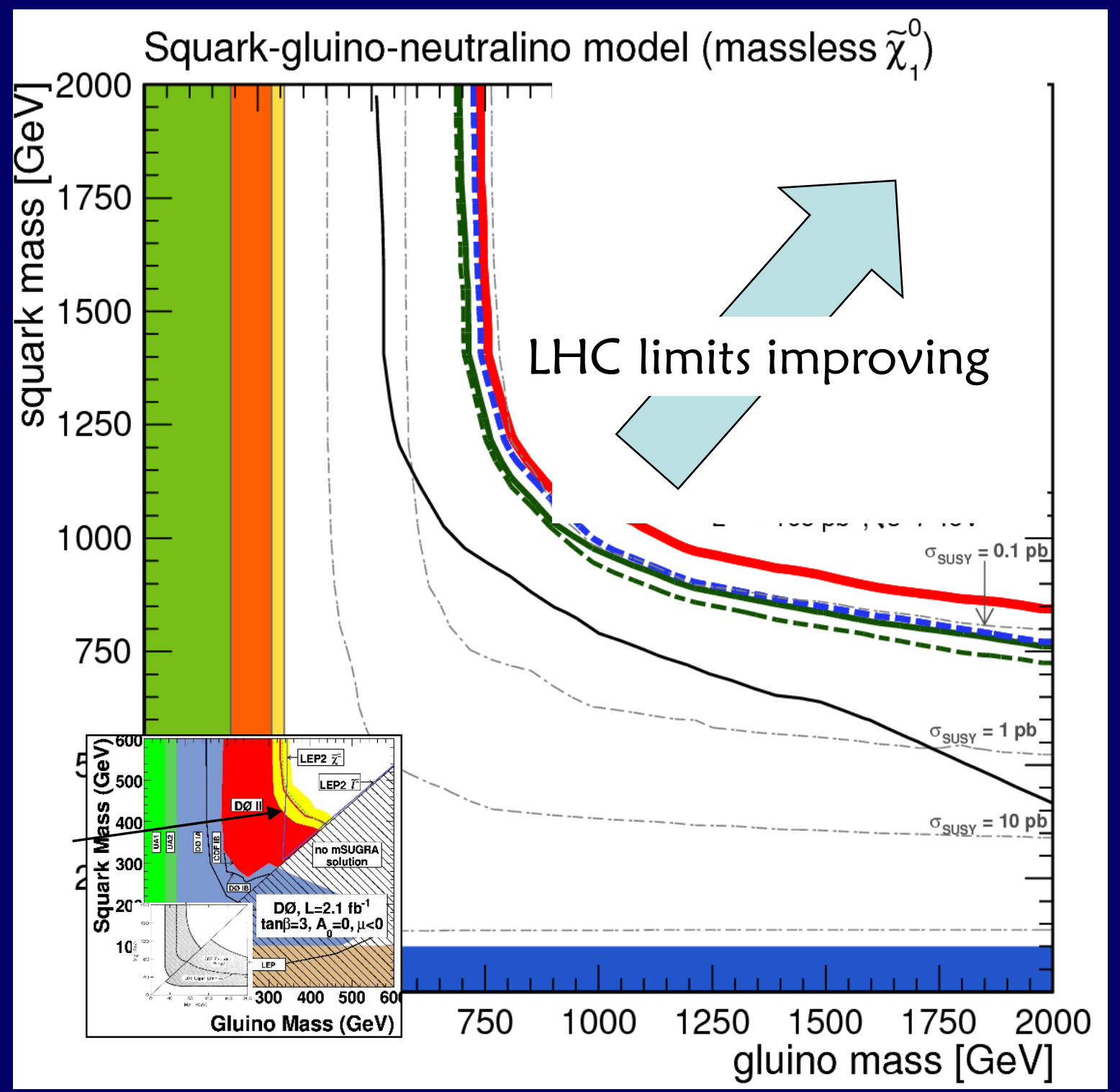




\section{Highlights - New phenomena}

LHC has overtaken Tevatron in more ways than one

"400 Physicists Fail to Find Supersymmetry" (NYTimes, ca 1992)

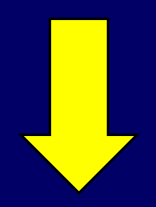

\section{“6000 Physicists Fail to} Find Supersymmetry"

(Tribume de (Bemede, 2018)

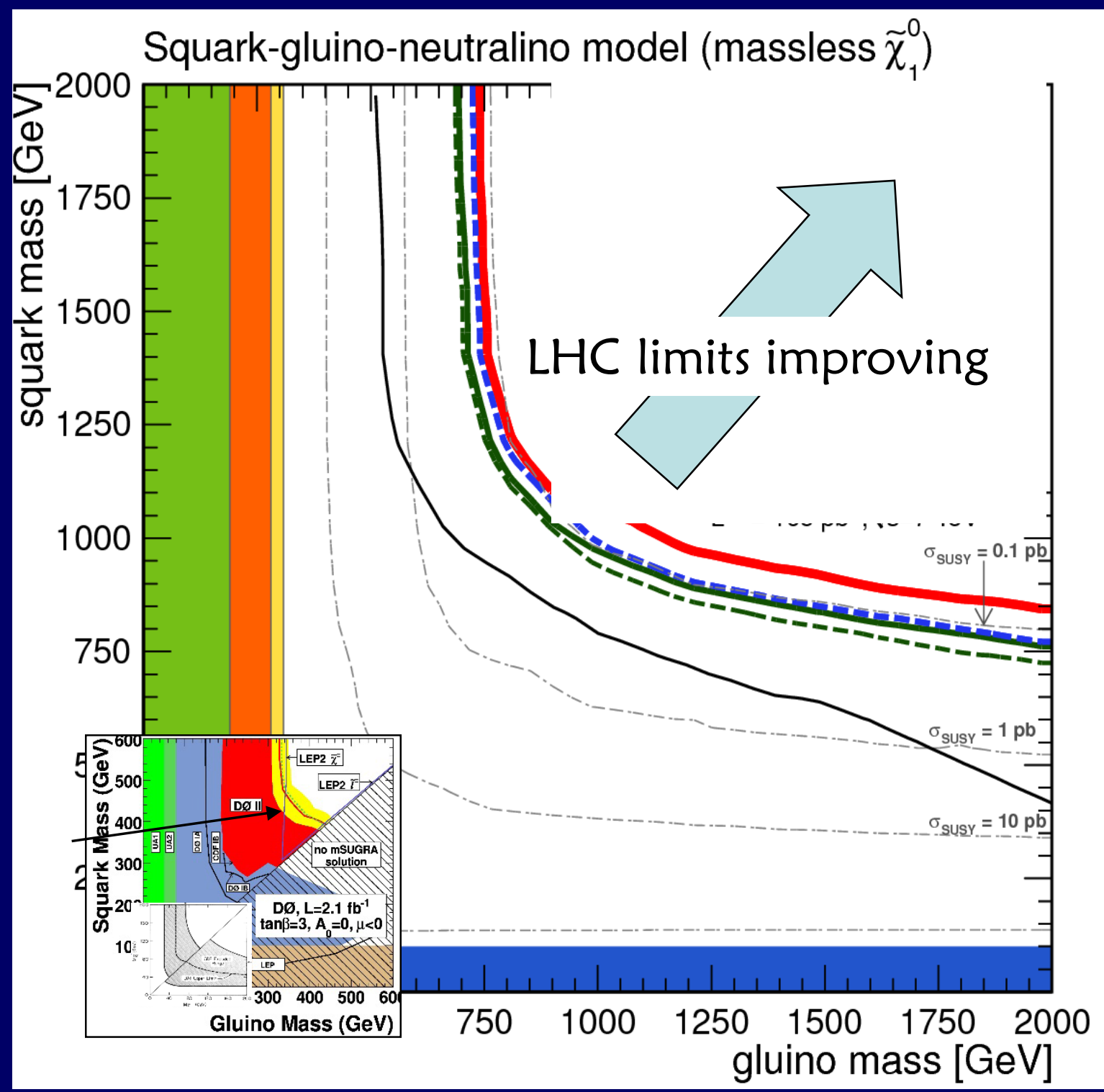




\section{The World of CDF and DO}
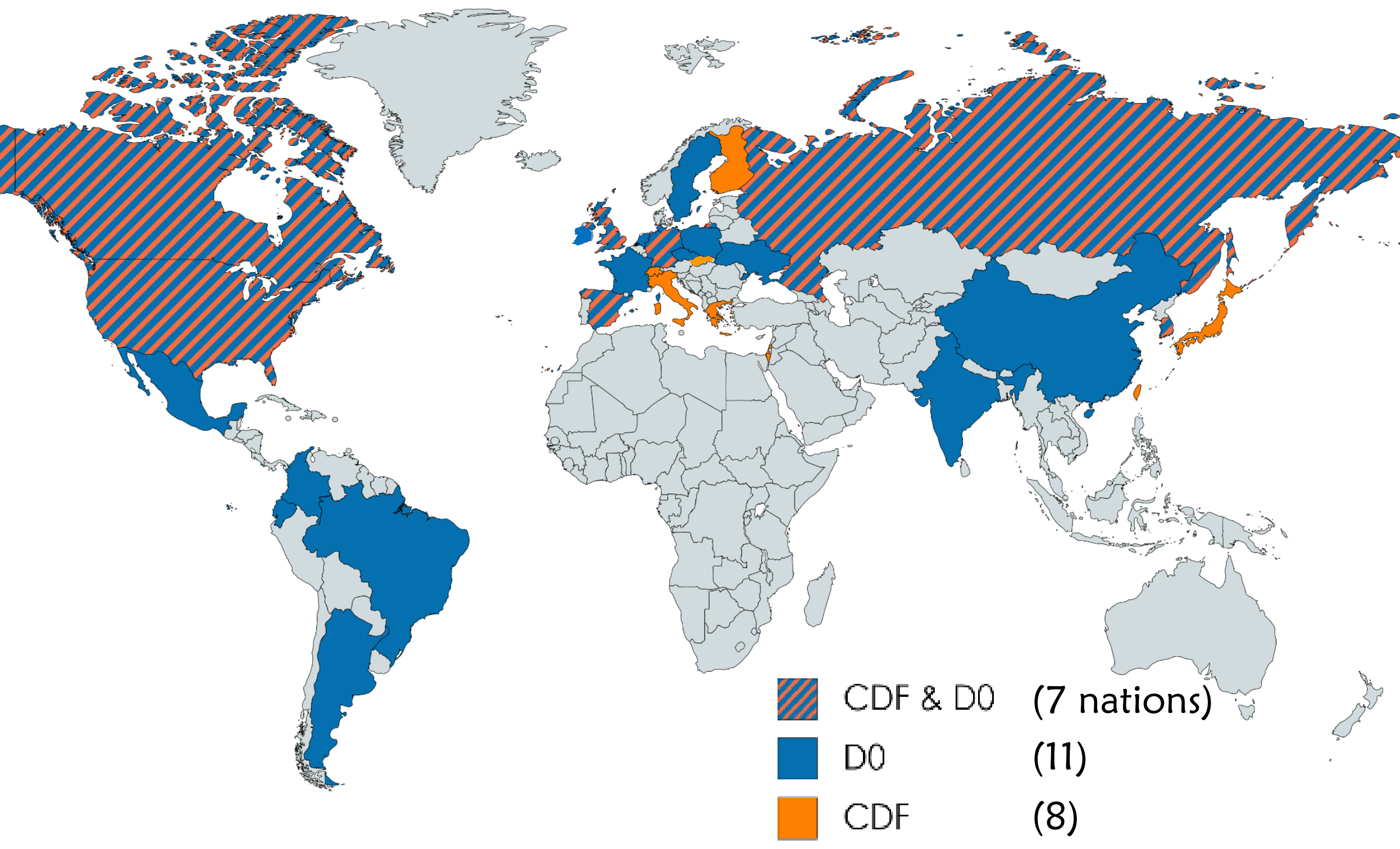


\section{The Tevatron by the Numbers}

40 years since inception of the Tevatron program (and still counting)

35 experiment-years of colliding beams delivered

Almost $12 \mathrm{fb}^{-1}$ delivered to CDF and DO (about $10 \mathrm{fb}^{-1}$ in analyses)

7 Accelerator Division Heads during the Tevatron era

$\sim 1200$ CDF and D0 publications; including 6 joint PRLs and 8 joint PRDs.

Almost 600 total Physical Review Letters

About 3000 collaborators over the lifetimes of the two experiments

\& $1128 \mathrm{PhD}$ theses

* 26 nations collaborated in CDF and DO (including 7 in both)

* 26 people served as spokespersons ( 3 did so twice)

\& About $2 \times 10^{15}$ antiprotons $(300 \mu \mathrm{C})$ died a horrible death at $\mathrm{B} \varnothing$ and $\mathrm{D} \varnothing$ 


\section{Summary}

The Tevatron experiments have provided a significant legacy in validating the Standard Model and dramatically increasing our knowledge of its particles and forces.

Their contributions still continue.
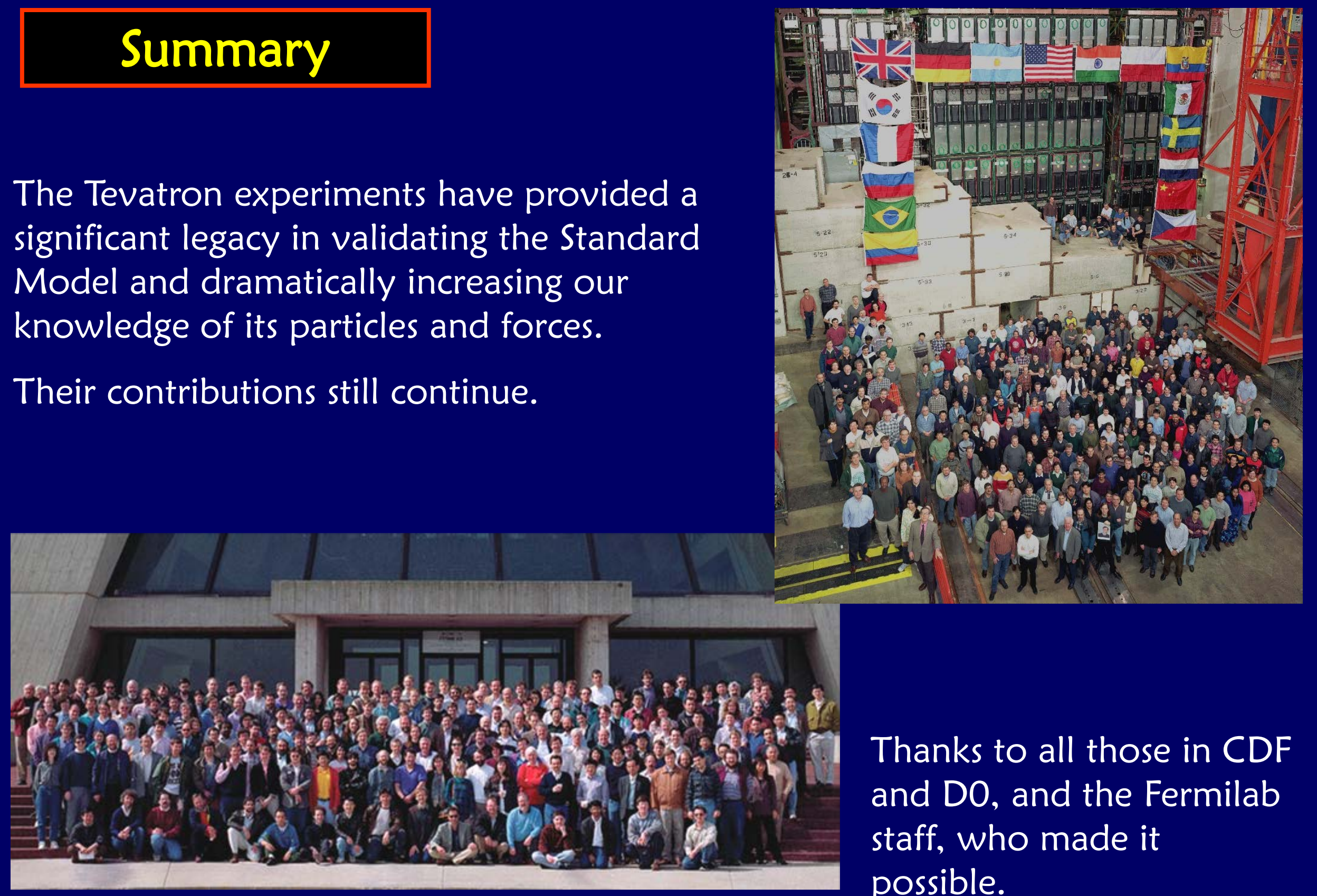

Thanks to all those in CDF and DO, and the Fermilab staff, who made it possible. 


\section{Thanks}
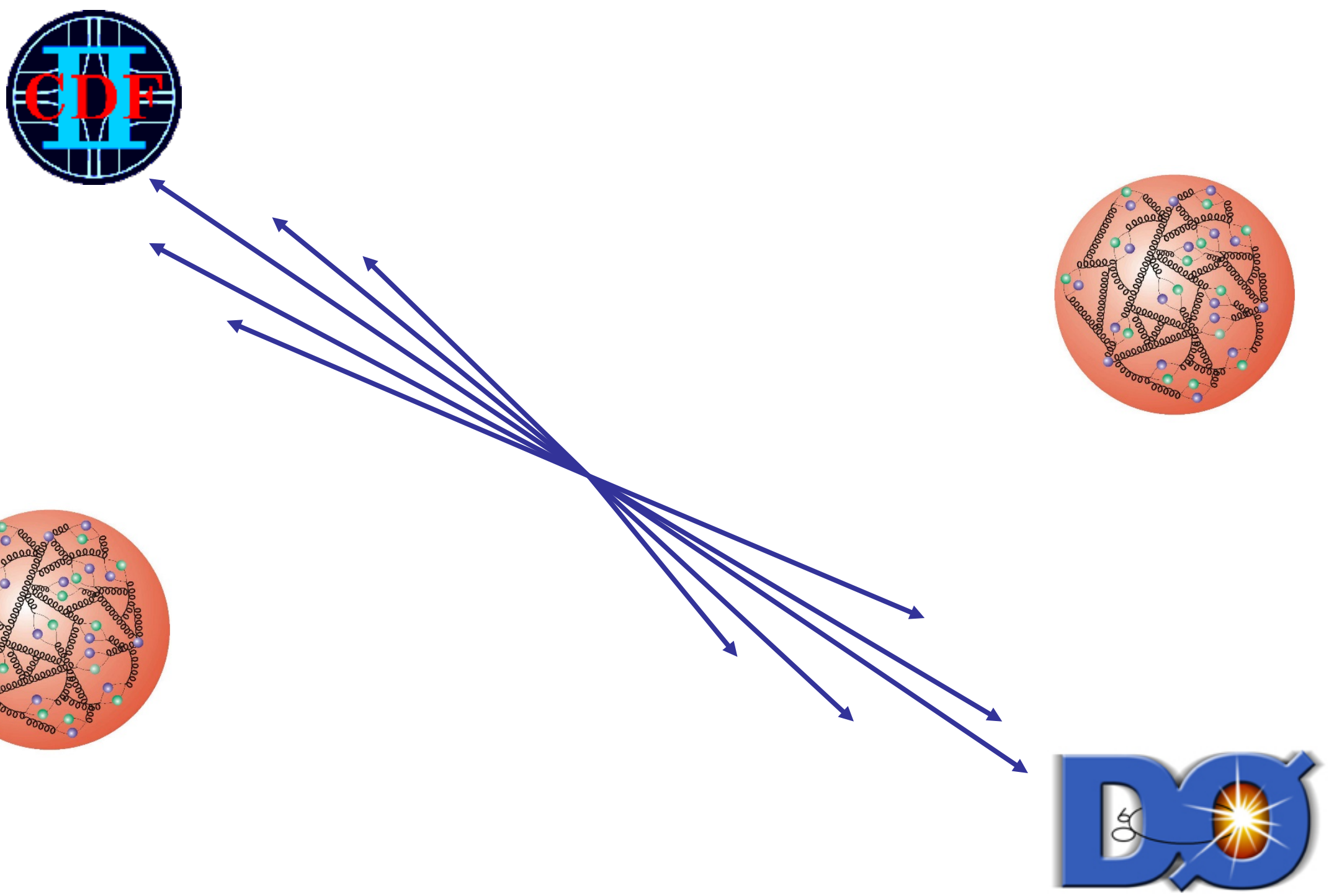